Supporting Information

\title{
Direct Fabrication of Discrete Large Functional Spherical Particles onto Organic Material Surfaces by Photografting Polymerization
}

\author{
Yongxin Wang, Yaowen Bai, Wenbin Zhong, Wenqing Huang, Wantai Yang*
}

State Key Laboratory of Chemical Resource Engineering, Beijing, 100029, P. R. China; Department of Polymer Science, Beijing University of Chemical Technology, Beijing, 100029, P.R. China.

\section{Experimental section}

\section{Materials}

Biaxially oriented polypropylene (BOPP) films with a diameter of $6 \mathrm{~cm}$ and a thickness of about $30 \mu \mathrm{m}$ were used as substrates after having been extracted with acetone for $36 \mathrm{~h}$ to remove additives and impurities. Methyl methacrylate (MMA) was purchased from Beijing Yili Chemical Co. Ltd., China, and purified by vacuum distillation before use. $N$ vinyl pyrrolidone (NVP) was obtained from the Chemical Plant of Nankai University. The initiator benzophenone (BP) and the cross-linking agent 1, 2-divinyl benzene (DVB) were obtained from Shanghai Chemical Reagents Co. Ltd., China. Hexadecyl trimethylammonium bromide (HTAB), acetone and methanol were obtained from Beijing Chemical Reagents Co. Ltd., China. All of the above-mentioned regents were used as received without any further treatments. Deionized water had a conductivity of $0.5 \mu \mathrm{s} \cdot \mathrm{cm}^{-}$ 1 
Preparation of the MMA/NVP microemulsion. The microemulsions was prepared at room temperature under vigorous stirring by adding MMA and DVB (molar ratio, 30:1) to aqueous NVP solutions containing $15 \mathrm{wt} \% \mathrm{HTAB}$.

Surface Photografting Polymerization. As a first step, $10 \mu \mathrm{l}$ of a BP acetone solution was sandwiched between two BOPP films. When the solvent was evaporated, $20 \mu \mathrm{l}$ MMA/ NVP microemulsion was added and spread into a liquid layer onto the above unit, which was then fixed between two quartz plates and irradiated under UV light (from a 1 $\mathrm{kW}$ high pressure mercury lamp) for 1-10 min at room temperature. Subsequently, the top and bottom films were split off and extracted with methanol for $6 \mathrm{~h}$ to remove the emulsifier. This step was followed by extraction with acetone for $6 \mathrm{~h}$ and with deionized water for $4 \mathrm{~h}$. The films were then oscillated through an ultrasound treatment to remove the unreacted BP, homopolymer and ungrafted particles. Following each step, the films were dried at room temperature to constant weight.

Synthesis of Ag/PMMA-PVP Composite Nanoparticles. The purified grafting films were immersed in a 10 wt \% aqueous $\mathrm{AgNO}_{3}$ solution overnight. Subsequently, the films were cleaned with deionized water and immersed in a $50 \mathrm{wt} \%$ aqueous hydrazine hydrate solution. Finally, the films were extracted with deionic water and oscillated through an ultrasound treatment to remove the homopolymers attached on the films.

Characterization. The topography of the films was observed with AFM (Nanoscope III, Digital Instruments) and SEM (S250 HK3). The EDS spectrum was obtained with a Cambridge XL-30, FEI. The characteristic groups of the films were detected by an FT-IR Spectrometer (Nicolet Nexus 670) equipped with variable angle horizontal ATR accessory, on which a $45^{\circ}$ rectangular ZnSe crystal was used. 

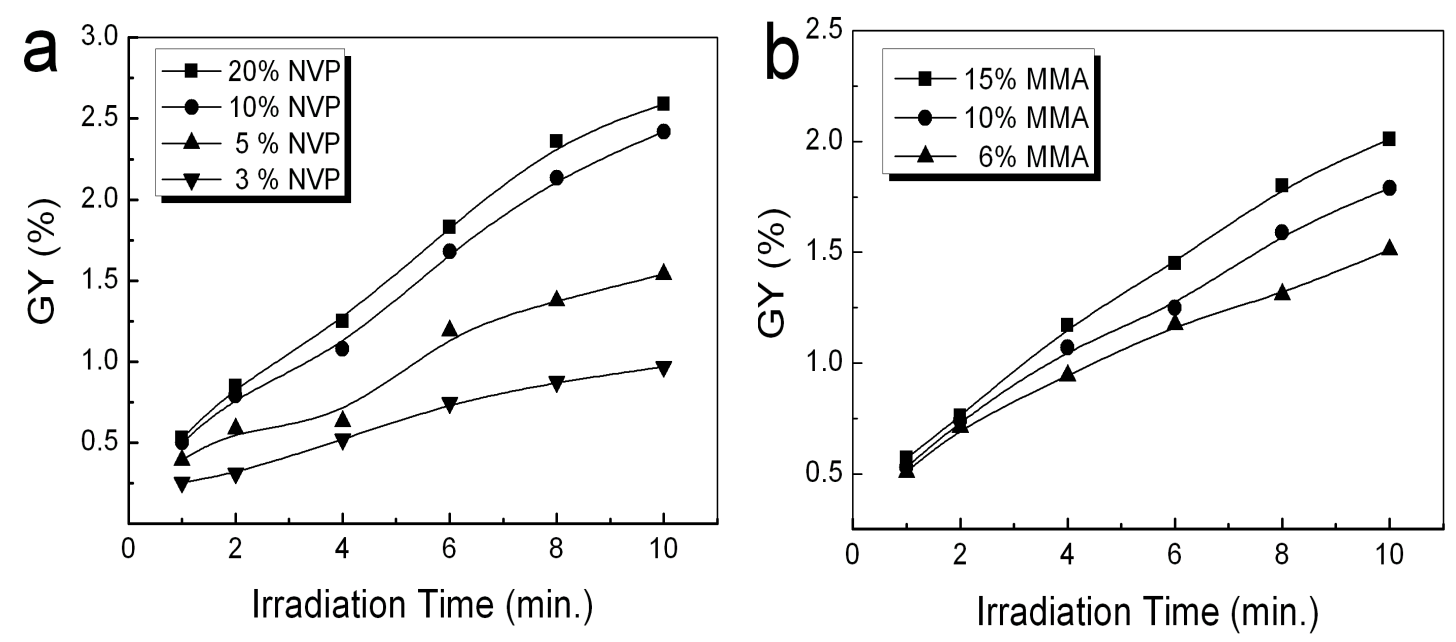

Figure S1. The relationship between surface grafting yields and irradiation time. The BP concentration was $5 \times 10^{-2} \mathrm{wt} \%$ at room temperature and the UV intensity was $7 \mathrm{~K} \mu \mathrm{w} / \mathrm{cm}^{2}$. a) MMA concentration was fixed at $20 \mathrm{wt} \%$; b) NVP concentration was fixed at $10 \mathrm{wt} \%$.

The surface grafting yields (GY) of the PP films were calculated by the equation:

$$
\mathrm{GY} \%=(\mathrm{Wg} / \mathrm{Wo}) \times 100 \%
$$

where Wo is the weight of the blank film; $\mathrm{Wg}$ is the weight of grafting polymers, equals the weight of washed film minus the weight of blank film.

In a previous report, ${ }^{12 b}$ the photografting of MMA microemulsion on the surface of PP films was described as high efficiency but low GY. However, the surface grafting yields have been greatly improved here. When the MMA concentration was set at $20 \mathrm{wt} \%$, the GY was in the range of $0.3-2.7 \%$, which corresponding to an average thickness range of 90-810 nm; when the NVP concentration was set at $10 \mathrm{wt} \%$, the GY was in the range of 0.5-2.0\%, which corresponding to an average thickness range of 150-600 nm. Moreover, when MMA concentration was given, the increase of NVP concentration showed a good help for the increase of GY (Figure S1 a). It is believed that the PVP chains helped more PMMA nanoparticles linked together and grafted on the surface of PP films, especially in 
the system with a high concentration of NVP. Figure S2 b may also provide a support. When the concentration of NVP was fixed in the microemulsion, the decrease of GY was gradual with the decrease of MMA concentration. Therefore, it may be thought that PVP chains preferred to help PMMA particles linking together in the current systems.
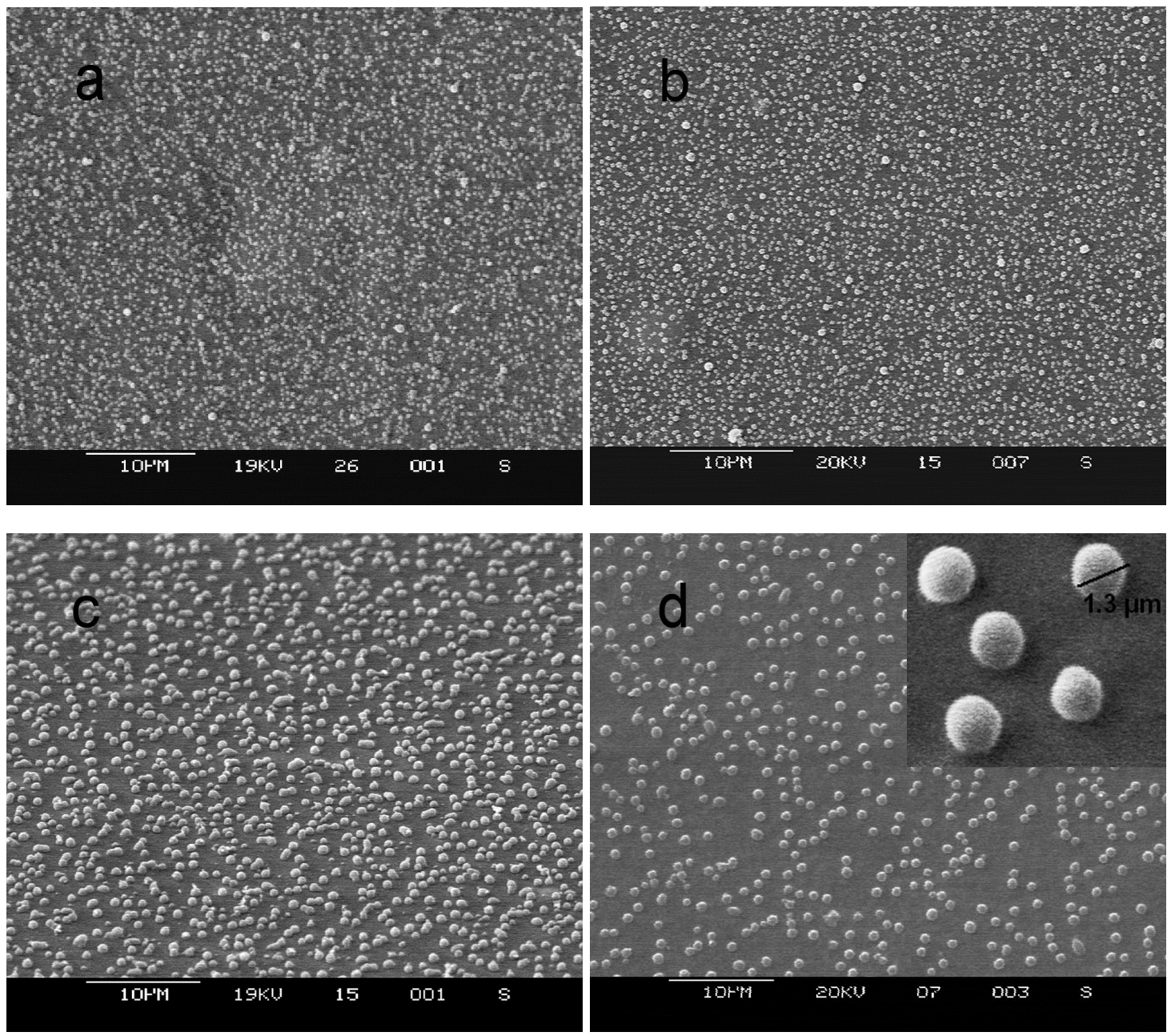

Figure S2 SEM images of the graft films with an MMA concentration of 20 wt $\%$ containing: a) $3 \mathrm{wt} \%$ NVP with particle diameters of $\sim 350 \mathrm{~nm}$; b) 5 wt $\%$ NVP with particle diameters of $\sim 550 \mathrm{~nm}$; c) $10 \mathrm{wt} \%$ NVP with particle diameters of $\sim 1 \mu \mathrm{m}$; d) 20 wt $\%$ NVP with particle diameters of $\sim 1.3 \mu \mathrm{m}$. 
The scanning electronic microscopy (SEM) images displayed in Figure S2 showed the typical surface morphology of the BOPP films after grafting with the MMA/NVP microemulsion. Numerous spherical particles in the sub-micron to microscale were tethered to the surface of the BOPP film as a monolayer. Moreover, the size of the particles changed with the mass ratio of MMA to NVP in the recipe. Throughout the experiments, the concentration of MMA was maintained at $20 \mathrm{wt} \%$. When the concentration of NVP in the system was low, i.e. $3 \mathrm{wt} \%$ and $5 \mathrm{wt} \%$, the diameters of the obtained particles were approximately $350 \mathrm{~nm}$ and $550 \mathrm{~nm}$, respectively. For NVP concentrations of $10 \mathrm{wt} \%$ and $20 \mathrm{wt} \%$, the particle sizes correspondingly increased to 1 $\mu \mathrm{m}$ and $1.3 \mu \mathrm{m}$, respectively. Meanwhile, these particles displayed narrow size distributions and perfect morphological shapes. It was believed that the increase in NVP concentration in the microemulsion not only promoted the grafting of the PMMA nanoparticles but also facilitated the assembly. As a result, the final grafting particles portrayed increasingly narrow size distributions.
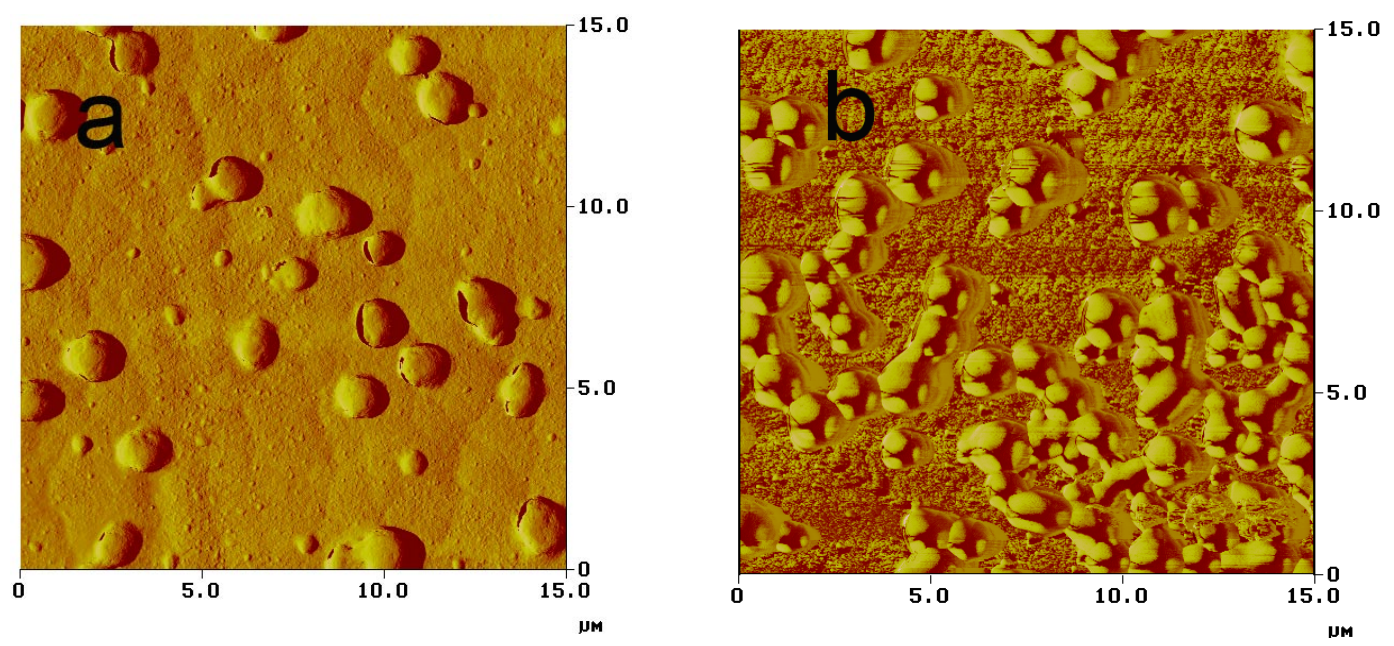

Figure S3 AFM images showing the hollow structures of grafted particles. The microemulsion containing $10 \mathrm{wt} \% \mathrm{NVP}$, the BP concentration $5 \times 10^{-2} \mathrm{wt} \%$, the UV 
intensity $7 \mathrm{~K} \mu \mathrm{W} / \mathrm{cm}^{2}$ and the irradiation time $10 \mathrm{~min}$ at room temperature: a) containing $15 \mathrm{wt} \%$ MMA and b) AFM image containing $10 \mathrm{wt} \%$ MMA.

In Figure S3, AFM images provide a large scale and high-magnified vision of the hollow particles. When the concentration of MMA kept in a higher level (c.a. $15 \mathrm{wt} \%$ ), the wall thickness of the obtained hollow particles was probably high, and just crevasses could be observed. When the concentration deceased to $10 \mathrm{wt} \%$, the wall thickness decreased correspondingly. The wall of particle was easily damaged under the pressure of AFM probe so that many crushed particles were observed.

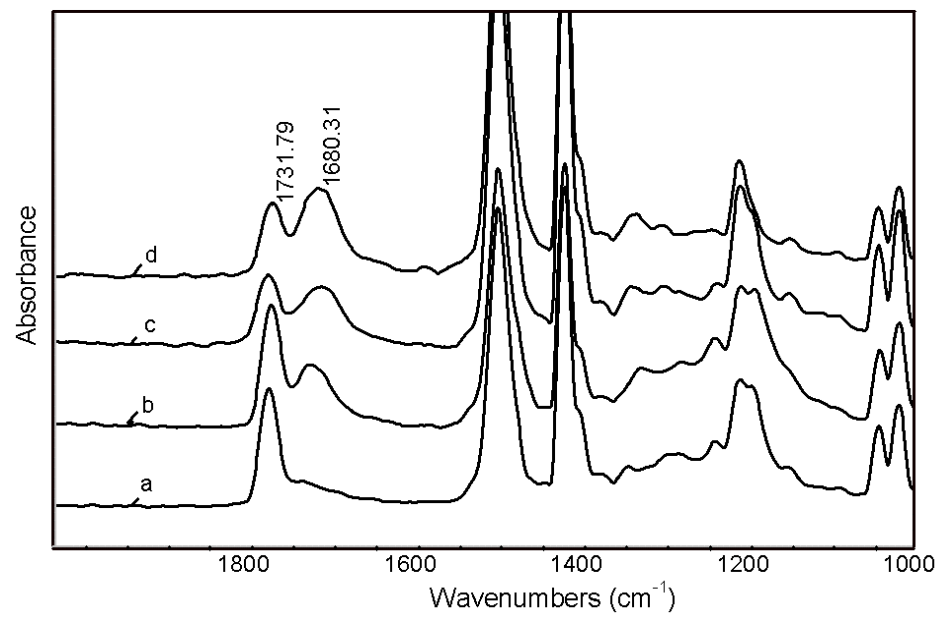

Figure S4. ATR-FTIR spectra of BOPP films grafted with MMA/NVP microemulsions. The BP concentration was $5 \times 10^{-2} \mathrm{wt} \%$ at room temperature and the UV intensity was 7 $\mathrm{K} \mu \mathrm{w} / \mathrm{cm}^{2}$. The original microemulsion contained a) $3 \mathrm{wt} \% \mathrm{NVP}$ and $20 \mathrm{wt} \% \mathrm{MMA}$; b) 5 wt $\%$ NVP and $20 \mathrm{wt} \%$ MMA; c) $20 \mathrm{wt} \%$ NVP and $20 \mathrm{wt} \%$ MMA; d) $20 \mathrm{wt} \%$ NVP and $10 \mathrm{wt} \%$ MMA.

Figure S4 shows the attenuated total reflection Fourier transform infrared (ATR-FTIR) spectra of BOPP films grafted with various concentrations of the MMA/NVP 
microemulsions. The absorption peak at $1731.79 \mathrm{~cm}^{-1}$ corresponded to $v(\mathrm{C}=\mathrm{O})$ of PMMA, and the peak at $1680.31 \mathrm{~cm}^{-1}$ to $\mathrm{v}(\mathrm{C}=\mathrm{O})$ of NVP. It should be noticed that the relative peak height at these two wavenumbers $\left(1731.79 \mathrm{~cm}^{-1}\right.$ to1680.31 $\left.\mathrm{cm}^{-1}\right)$ changed with the mass ratio of MMA/NVP in the recipe. With $3 \mathrm{wt} \% \mathrm{NVP}$ and $20 \mathrm{wt} \% \mathrm{MMA}$, the relatively small amount of NVP resulted in its adsorption being too weak to be observed. With an increasing NVP concentration in the recipe, the peak became more and more visible. When NVP was increased to $20 \mathrm{wt} \%$ and MMA decreased to $10 \mathrm{wt} \%$, it was not only easy to distinguish the dual peaks, but the peak-strength of the acyl-carbonyl group (belonging to PVP) was even higher than that of the ester-carbonyl group (belonging to PMMA).

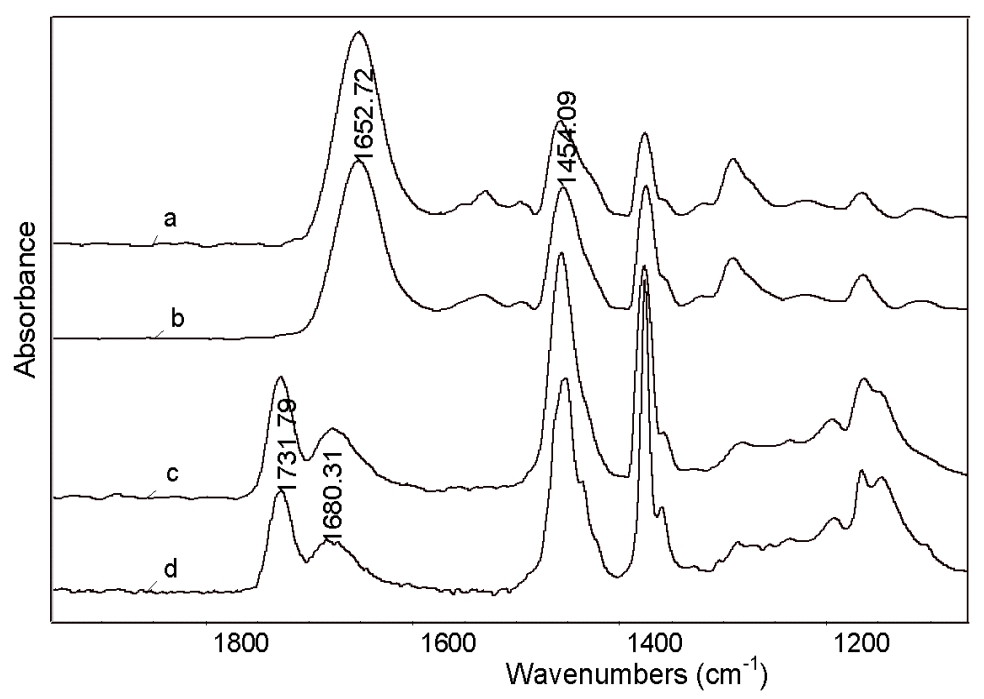

Figure S5. ATR-FTIR spectra of the BOPP films grafted with a $20 \mathrm{wt} \%$ NVP ethanol solution (a and b) and with a MMA/NVP (20 wt/10 wt $\%$ ) microemulsion (c and d). The a, c traces represent an ATR scanning angle of $35^{\circ}$ whereas for $b, d$ the ATR scanning angle was $50^{\circ}$. 
Furthermore, the role of PVP in the formation of the grafting particles can probably be understood from Figure S5. In the ATR-FTIR characterization, the grafting films have been scanned in two angles. Changing the scanning angle allows the measurements to be performed at different depths of the film surface and thus the grafting status could be illuminated. As can be seen in Figure S5, the strength ratio of the peaks at $1652.72 \mathrm{~cm}^{-1}$ ( $\mathrm{C}=\mathrm{O}$ expansion vibration of $\mathrm{PVP})$ to $1454.09 \mathrm{~cm}^{-1}\left(-\mathrm{CH}_{3}\right.$ deformation vibration of BOPP) was analyzed for each spectrum. As a comparison, the FTIR traces of the films grafted with an NVP ethanol solution are given in Figures S5 a and b. When the scanning angle was tuned from $35^{\circ}$ to $50^{\circ}$, the strength ratio displayed a notable decrease in a and b. However, this change was unnoticed for the films grafted with PVP/PMMA (as seen in c and d). As we know, the graft chains on the polymer surface have a tendency to move down the subsurface through conformational inversion. The PVP chains grafted on the surface by the solution method had little obstacle for their conversion, whereas those chains comprised in the surface grafting particles were hindered by the steric influence of the particles. These results suggest that the PVP chains participated in the formation of the surface grafting supramolecular particles. 